\title{
Efficient mucosal vaccination mediated by the neonatal Fc receptor
}

\author{
Lilin Ye ${ }^{1}$, Rongyu Zeng ${ }^{1}$, Yu Bai ${ }^{1}$, Derry C Roopenian ${ }^{2} \&$ Xiaoping Zhu ${ }^{1,3}$
}

\begin{abstract}
Almost all infectious diseases are initiated at mucosal surfaces, yet intramuscular or subcutaneous vaccination usually provides only minimal protection at sites of infection owing to suboptimal activation of the mucosal immune system. The neonatal Fc receptor (FcRn) mediates the transport of IgG across polarized epithelial cells lining mucosal surfaces. We mimicked this process by fusing a model antigen, herpes simplex virus type-2 (HSV-2) glycoprotein gD, to an IgG Fc fragment. Intranasal immunization, together with the adjuvant $\mathrm{CpG}$, completely protected wild-type, but not FcRn knockout, mice after intravaginal challenge with virulent HSV-2 186. This immunization strategy induced efficient mucosal and systemic antibody, B- and T-cell immune responses, with stable protection for at least 6 months after vaccination in most of the immunized animals. The FcRn-IgG transcellular transport pathway may provide a general delivery route for subunit vaccines against many mucosal pathogens.
\end{abstract}

Vaccines that protect against infectious agents entering the body at mucosal surfaces must induce both mucosal and systemic immune responses to cope with early infection and pathogen spread ${ }^{1-4}$. The close association between mucosal epithelial cells and the immune effector cells within the laminar propria ${ }^{1-3}$ suggests that delivery of immunogens through the mucosal surface would be an ideal approach to achieve mucosal and, potentially, systemic immunity. However, the intercellular tight junctions of epithelial monolayers lining the mucosal surfaces render them impervious to macromolecule diffusion ${ }^{5}$. One approach to circumvent this problem involves targeting mucosal vaccines onto differentiated microfold (sometimes called ' $M$ ') cells that punctuate the mucosal epithelium ${ }^{6}$. However, because columnar epithelial cells make up the great majority of mucosal surfaces, alternative mucosal vaccine delivery strategies that target these abundant epithelial cells may increase the efficacy of mucosal vaccines.

FcRn allows fetuses or newborns to obtain maternal IgG through the placental or intestinal route 7,8 . However, the receptor can also transport IgG antibody across mucosal surfaces in adult life ${ }^{9-12}$ and confer resistance to intestinal pathogens ${ }^{12}$. The observation that FcRn can transport IgG across mucosal epithelia suggested to us that fusion of an antigen to IgG Fc may enable FcRn-mediated transport of the antigen across the mucosal barrier. To test this possibility, we worked with the model pathogen HSV-2, which initiates infection primarily at the mucosa of the genital tract ${ }^{4}$. The model antigen, HSV-2 gD, is important in early viral infection and a target for both humoral and cellular immunity. We determined the ability of FcRn to deliver a fusion of $\mathrm{gD}$ to the $\mathrm{Fc}$ fragment across the respiratory mucosal barrier and then further defined the protective immune responses and mechanisms relevant to this mode of mucosal vaccine delivery.

To target $\mathrm{gD}$ for FcRn-mediated uptake, we first generated the fusion protein, $\mathrm{gD}-\mathrm{Fc} / \mathrm{wt}$, by cloning the extracellular domain of HSV-2 gD in frame with a modified form of the mouse IgG2a Fc fragment (Supplementary Fig. 1). We also generated a similarly modified $\mathrm{gD}-\mathrm{Fc} /$ mut fusion protein, which cannot bind FcRn owing to $\mathrm{H} \rightarrow \mathrm{A}$ substitutions at positions 310 and 433 (ref. 13). In both cases, the complement C1q-binding motif was eliminated to abrogate $\mathrm{C} 1 \mathrm{q}$ binding $^{14}$ (Supplementary Fig. 1a). To ascertain whether the gD-Fc/wt, but not the $\mathrm{gD}-\mathrm{Fc} / \mathrm{mut}$, fusion proteins were transported by $\mathrm{FcRn}$, we assayed for FcRn-dependent transcytosis of intact $\mathrm{gD}$-Fc/wt in inner medullary collecting duct cells expressing FcRn ${ }^{15}$ (Supplementary Fig. 2a). We then determined whether the gD-Fc/wt reached the bloodstream after intranasal inoculation. We confirmed FcRn expression in mouse trachea and lung and its absence in the adult intestine (Supplementary Fig. 2b). After intranasal (i.n.) administration of $20 \mu \mathrm{g}$ of either $\mathrm{gD}, \mathrm{gD}-\mathrm{Fc} /$ mut or $\mathrm{gD}-\mathrm{Fc} / \mathrm{wt}$ protein in FcRn WT and FcRn knockout mice ${ }^{16}$, we noted substantial gD-Fc/wt accumulation in the sera of FcRn WT mice only (Supplementary Fig. 2c). These results showed that efficient delivery of $\mathrm{gD}$ across the respiratory barrier to the bloodstream was dependent on the Fc moiety and its ability to interact with FcRn.

Engagement of FcRn greatly increased the efficiency by which gD antigens induced antigen-specific antibody and cellular immune responses. We then determined whether FcRn-dependent transport augmented the potential of the gD protein to mount an immune response. Mice were immunized i.n. with gD-Fc, gD protein or PBS, all in combination with $\mathrm{CpG}$, and received boosters after 2 weeks. The free $\mathrm{gD}$ protein, not linked to an Fc fragment, allowed us to both evaluate FcRn-independent effects in vivo and determine the magnitude of any observed enhancement in immune responses conferred by fusion to $\mathrm{Fc}$ and recognition by $\mathrm{FcRn}$. Because at least some immature dendritic cells are immunologically tolerant in vivo, we co-administered CpG, a ligand for Toll-like receptor 9, in an effort to overcome possible mucosal tolerance ${ }^{17}$. In a different study, we found that co-administration of $\mathrm{CpG}$ oligonucleotides with an Fc fusion

${ }^{1}$ Laboratory of Immunology, Virginia-Maryland Regional College of Veterinary Medicine, University of Maryland, College Park, Maryland, USA. ${ }^{2}$ The Jackson Laboratory, Bar Harbor, Maine, USA. ${ }^{3}$ Maryland Pathogen Research Institute, University of Maryland, College Park, Maryland, USA. Correspondence should be addressed to X.Z. (xzhu1@umd.edu). 
a

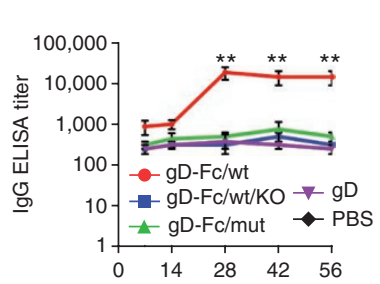

Days after primary immunization b

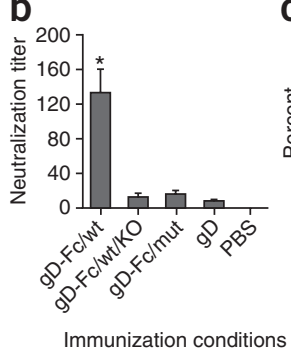

C

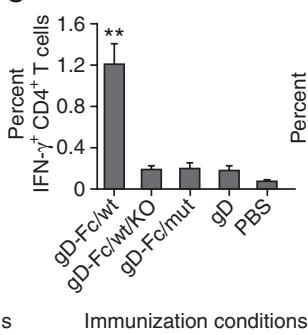

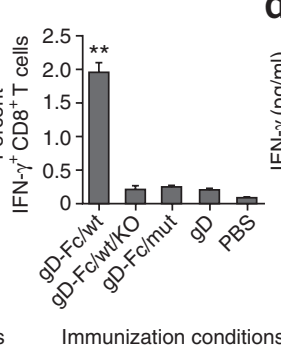
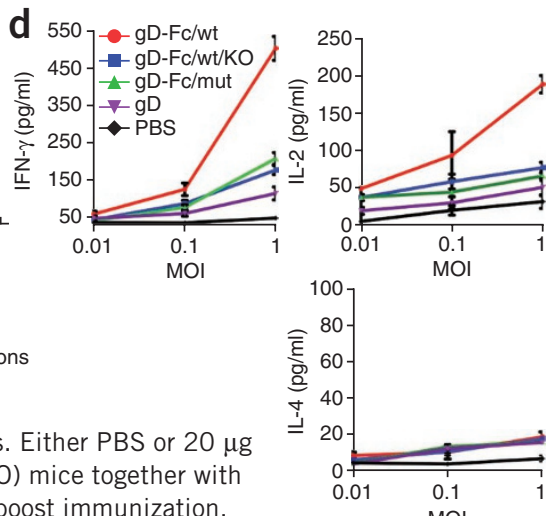

Figure 1 FcRn-targeted mucosal vaccination induces enhanced gD-specific antibody and T-cell responses. Either PBS or $20 \mu \mathrm{g}$ of gD-Fc/wt, gD-Fc/wt/KO, gD-Fc/mut or gD were administered i.n. to wild-type (WT) or FcRn knockout (KO) mice together with $20 \mu \mathrm{g}$ CpG. (a) Measurement of anti-HSV-2 gD-specific IgG antibody titers in serum before and after the boost immunization. Anti-HSV-2 gD-specific IgG antibody at the indicated days was measured in serum by ELISA. Immunization conditions are indicated on the right. The curves represent mean values for each group ( \pm s.e.m.). Black line for PBS is overlapped with $x$ axis due to negative lgG titer in PBS. Values marked with asterisk in this and subsequent figures: ${ }^{*}, P<0.05$; ${ }^{*}, P<0.01$. (b) Test of neutralizing activity in the immunized sera. Sera were heat inactivated, diluted tenfold, then in twofold steps in MEM with $2 \%$ FBS. HSV-2 (50 p.f.u.) was added and incubated at $37^{\circ} \mathrm{C}$ for $1 \mathrm{~h}$. Finally, the mixture was removed and washed, overlaid with $0.8 \%$ methylcellulose in $2 \%$ FBS containing DMEM and further incubated for $72 \mathrm{~h}$ at $37{ }^{\circ} \mathrm{C}$. The titers were expressed as the reciprocal of the twofold serial dilution preventing the appearance of the cytopathic effects (CPE) over control sera. Each assay was done in triplicate. (c) The percentage of IFN- $\gamma$-producing T cells in the spleen $4 \mathrm{~d}$ after the boost. Spleen cells from the immunized mice were stimulated for $10 \mathrm{~h}$ with purified gD or medium control. Lymphocytes were gated by forward and side scatter and T cells labeled with anti-CD3 and identified by their respective surface markers CD4 and CD8 and intracellular IFN- $\gamma$ staining. Immunization conditions are displayed on the bottom. Numbers represent the percentage of IFN- $\gamma^{+} \mathrm{CD}^{+} \mathrm{CD}^{+}$(left panel) or IFN- $\gamma^{+} \mathrm{CD}^{+} \mathrm{CD}^{+}$(right panel) T cells. Isotype controls included FITC-mouseIgG1 with baseline response. (d) Cytokine secretions from the stimulated spleen T cells. Spleen cells were collected on day 4 after the boost. Cells were stimulated in vitro specifically with different multiplicities of infection (MOI) of inactivated HSV-2 virus as indicated for $24 \mathrm{~h}$. Cytokines IFN- $\gamma$, IL-2 and IL-4 in the culture supernatant were detected by ELISA. Data are representative of three experiments with three immunized mice pooled in each group.

protein was required to induce a robust IgG response (unpublished data). Significantly $(P<0.01)$ higher titers of IgG were seen in the gD-Fc/wt-immunized mice when compared with the gD-, gD-Fc/mut-, gD-Fc/wt/KO-immunized and PBS-treated groups (Fig. 1a). Moreover, sera from the $\mathrm{gD}-\mathrm{Fc} / \mathrm{wt}$-immunized mice exhibited strong neutralizing activity (Fig. 1b) and after passive transfer, protected animals from intravaginal challenge more efficiently than sera from all other groups (Supplementary Fig. 3). Likewise, mice immunized with $\mathrm{gD}-\mathrm{Fc} / \mathrm{wt}$ had a significantly higher frequency $(P<0.01)$ of IFN- $\gamma$-producing $\mathrm{CD}^{+}$and $\mathrm{CD}^{+}$splenic $\mathrm{T}$ cells in response to gD stimulation, relative to the other groups (Fig. 1c). The cytokine responses were dominated by IFN- $\gamma$ and interleukin (IL)-2; there was little IL-4 production (Fig. 1d). The existence of this dominant T-helper-1 cell cytokine response was also supported by a major IgG2a subclass in the sera of the immunized mice (Supplementary Fig. 4). It remains uncertain whether this polarized T-cell response was caused by mucosal immunization by means of FcRn targeting or, more likely, by the CpG used as adjuvant ${ }^{17}$.

That FcRn-targeted mucosal delivery of subunit vaccine elicited a strong mucosal immune response is supported by four lines of evidence.

First, to ascertain the ability of the FcRn-targeted mucosal immunization to induce local humoral immune responses, we monitored the activated germinal center reaction in the mediastinal lymph nodes (MeLNs) and spleens $10 \mathrm{~d}$ after the boost, as the nasopharynxassociated lymphoid tissue and MeLNs that drain the lungs are where mucosal immune responses are initiated against i.n. administered antigens ${ }^{18}$. Activated germinal centers are characterized by the presence of peanut agglutinin (PNA)-positive areas and highlevel expression of FAS apoptotic death receptor in activated $\mathrm{B}$ cells $\left(\mathrm{B} 220^{+}\right)^{19}$. The gD-Fc/wt immunization induced a substantially higher percentage of $\mathrm{FAS}^{+} \mathrm{PNA}^{+} \mathrm{B} 220^{+} \mathrm{B}$ cells in the MeLN or spleen in comparison with that of other groups (Fig. 2a), which was further validated by immunofluorescence imaging (Fig. 2b). In addition, the germinal center structure in the MeLNs in WT mice immunized by $\mathrm{gD}-\mathrm{Fc} / \mathrm{wt}$ was sustained much longer in comparison with that of other groups (Fig. 2c). Administration of $\mathrm{CpG}$ alone failed to elicit appreciable formation of germinal centers in the draining MeLNs, spleens or lungs (Supplementary Fig. 5). Because the formation and maintenance of germinal centers generally lead to the differentiation of memory B cells and long-lived plasma cells, we also examined the mesenteric, cervical and inguinal lymph nodes. We did not detect germinal center formation or a significant number of IFN- $\gamma$-secreting T cells at those sites.

Second, it has been shown that i.n. administered antigens can induce bronchus-associated lymphoid tissue (iBALT) in the lung, which is similar to germinal center structures ${ }^{19}$. Such germinal center-like structures could be detected in the lungs of gD-Fc/wt-, but not gD-Fc/mut-immunized mice or other groups of immunized animals (Fig. 2d and Supplementary Fig. 5). The germinal centerlike iBALT in the lung was most likely induced locally because of the presence of the transported gD antigen in the MeLNs and lung.

Third, to assess the ability of FcRn-targeted mucosal immunization to induce gD-specific antibody in mucosal secretions, we collected bronchial alveolar lavage (BAL) and vaginal washings $10 \mathrm{~d}$ after the boost and analyzed both for gD-specific IgG and IgA by enzymelinked immunosorbent assay (ELISA). Significantly $(P<0.01)$ increased levels of gD-specific IgG were present in the BAL and vaginal washings (Supplementary Fig. 6) of the gD-Fc/wt-immunized mice. WT, but not FcRn knockout, mice that received gD-Fc/wt had high levels of gD-specific IgG in BAL and vaginal washings $(P<0.01$; Supplementary Fig. 6), suggesting that the induction of mucosal IgG is mediated by $\mathrm{FcRn}$. IgG levels in BAL and vaginal washings were much higher than levels of IgA (Supplementary Fig. 6).

Fourth, with respect to $\mathrm{T}$-cell immune responses in the lung and MeLNs, $4 \mathrm{~d}$ after the boost we detected significantly $(P<0.01)$ more IFN- $\gamma$-producing $\mathrm{CD}^{+}$and $\mathrm{CD}^{+} \mathrm{T}$ cells (Fig. 2e,f) in response to $\mathrm{gD}$ stimulation in mice immunized with the $\mathrm{gD}-\mathrm{Fc} / \mathrm{wt}$. We conclude that the combination of $\mathrm{CpG}$ with antigen targeting to 

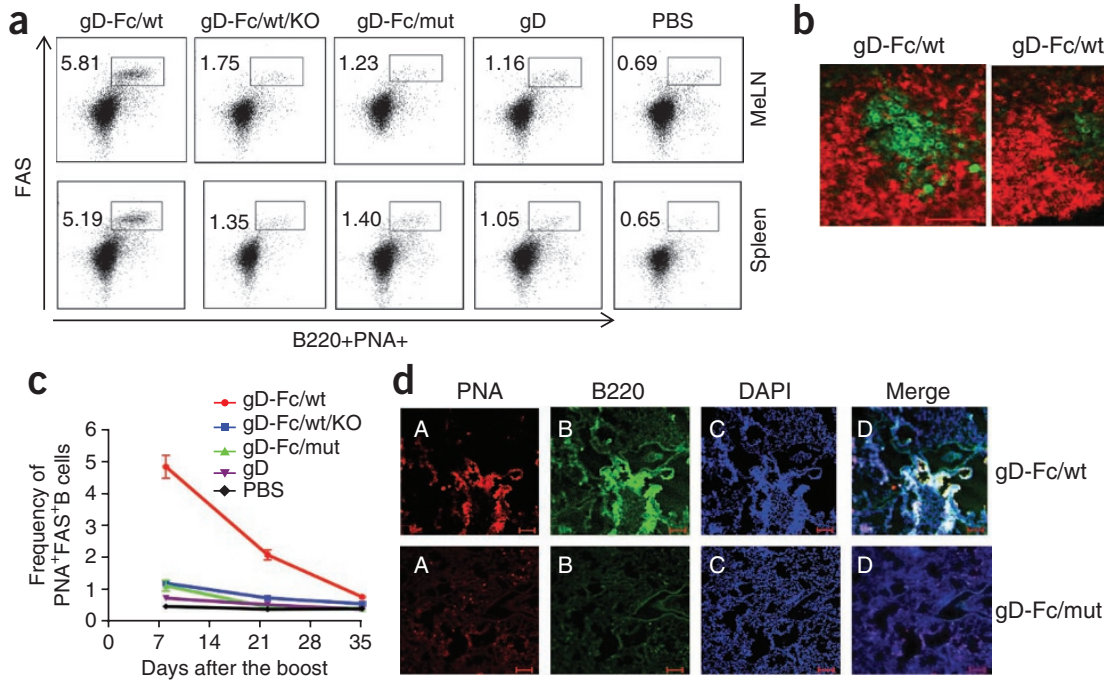

Figure 2 Local immune responses induced by FcRn-targeted mucosal immunization.

(a) Accumulation of activated $B$ cells in germinal centers in the mediastinal lymph nodes $(\mathrm{MeLN})$ and spleen. Representative flow cytometric analyses of germinal center $B$ cells among $\mathrm{CD} 19^{+} \mathrm{B} 22 \mathrm{O}^{+} \mathrm{B}$ cells in the MeLNs and spleen $10 \mathrm{~d}$ after the boost. B220+ PNA ${ }^{\text {high }}$ cells are $B$ cells that exhibit the phenotypic attributes of germinal center B cells. The germinal center staining in spleen was used as a positive control. Numbers are the percentage of activated germinal center B cells (PNA ${ }^{+}$FAS $\left.^{+}\right)$among gated B cells and are representative of three independent experiments. (b) Germinal center formation and presence of activated B cells following immunizations as indicated. Frozen MeLN sections at day 10 from the immunized mice were co-stained with biotin-PNA (developed with avidin-FITC) and Alexa647 labeled anti-lgD. Scale bars represent $50 \mu \mathrm{m}$. (c) Quantitative analysis of germinal centers following immunization. The dynamics of the frequency of germinal center $\mathrm{B}$ cells $\left(\mathrm{FAS}^{+} \mathrm{PNA}^{+}\right.$, gated on $\mathrm{CD} 19^{+} \mathrm{B} 220^{+}$cells) were plotted on days 10,22 and 35 after the boost. Data indicate the mean and S.E.M., $n=5$ mice. (d) The formation of inducible bronchus-associated lymphoid tissue (iBALT). Frozen serial sections of the lung were stained with biotin-PNA (germinal center, red) and anti-B220 (B cells, green), followed by Alexa 488-conjugated IgG of corresponding species and Alexa 555-Avidin. The nucleus is stained with DAPI (blue). A germinal center-like structure is shown in the merged panel by the white color. The data are representative of sections from at least three independent mice. Images were originally obtained at 10x magnification. Scale bars, $100 \mu \mathrm{m}$. (e,f). Presence of HSV-2 gD-specific T lymphocytes in the lung (e) and MeLNs (f). Lung or MeLN cells were collected from mice $4 \mathrm{~d}$ after the boost. Lymphocytes were gated based on their forward scatter (FSC) versus side scatter (SSC) profile. Intracellular staining for IFN- $\gamma$ was performed after surface staining of CD4 and CD8 molecules. The profiles shown are representative of five mice from three separate experiments. Numbers indicate percentages of IFN- $\gamma$-producing $\mathrm{T}$ lymphocytes from gated $\mathrm{CD} 4^{+}$and $\mathrm{CD} 8^{+} \mathrm{T}$ cells.

FcRn produced strong antibody as well as T-cell mucosal immune responses. An efficient protective vaccine must induce immunity in the mucosa to block viral entry. FcRn-targeted mucosal immunization may provide an efficient approach for the development of such protective mucosal vaccines.

In addition to humoral and cell-mediated immunity at the mucosal delivery site, an effective mucosal subunit vaccine should also elicit

Figure 3 FcRn-targeted mucosal immunization provides protective immunity to intravaginal challenge with virulent HSV-2 186. (a) Mean survival following genital HSV-2 challenge. Four weeks after the immunization, groups of five mice were challenged intravaginally with $1 \times 10^{4}$ p.f.u. of HSV-2 strain 186 . Percentage survival on the indicated days is calculated as the number of mice surviving divided by the number of mice in each group and represented three similar experiments. (b) Mean of viral titers following HSV-2 challenge. Virus titers were measured from vaginal washes by taking swabs on the indicated days after HSV-2 inoculation based on a plaque assay on Vero cell monolayers. (c) Increased presence of HSV-specific T Iymphocytes in the vaginal epithelium after challenge. Lymphocytes were harvested from collagenasedigested vaginal tissues $4 \mathrm{~d}$ after intravaginal inoculation with virus. Intracellular staining for IFN- $\gamma$ expression on $\mathrm{CD}^{+}{ }^{+}$and $\mathrm{CD}^{+}{ }^{+} \mathrm{T}$ cells was analyzed after gating on viable CD3+ lymphocytes. The numbers in each column show the percentage of IFN- $\gamma$-positive $\mathrm{T}$ lymphocytes from the gated $\mathrm{CD}^{+}{ }^{+}$or $\mathrm{CD} 8^{+} \mathrm{T}$ cells. Data shown are representative of three experiments, using three mice per experiment. immunity in systemic compartments that can access mucosal tissues distant from the immunization site ${ }^{1-3}$. Studies have found that mucosal immunization using the intranasal route is effective for generating antibody and $\mathrm{T}$-cell immune responses in the female
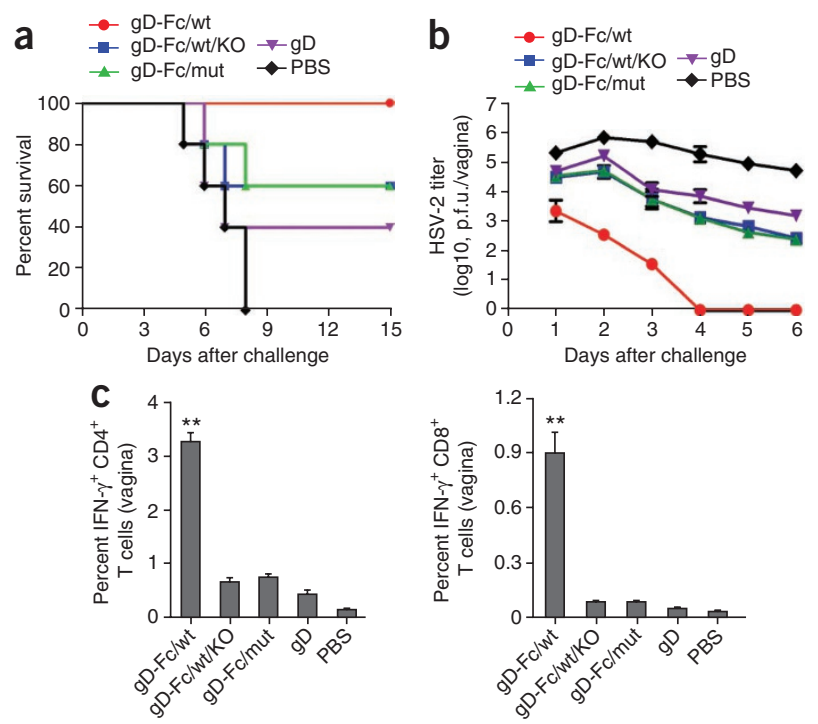
a

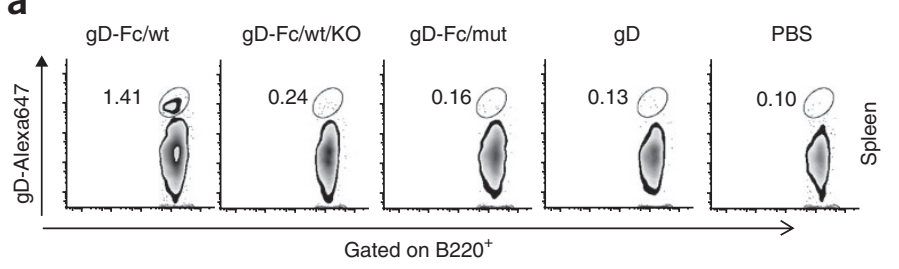

d

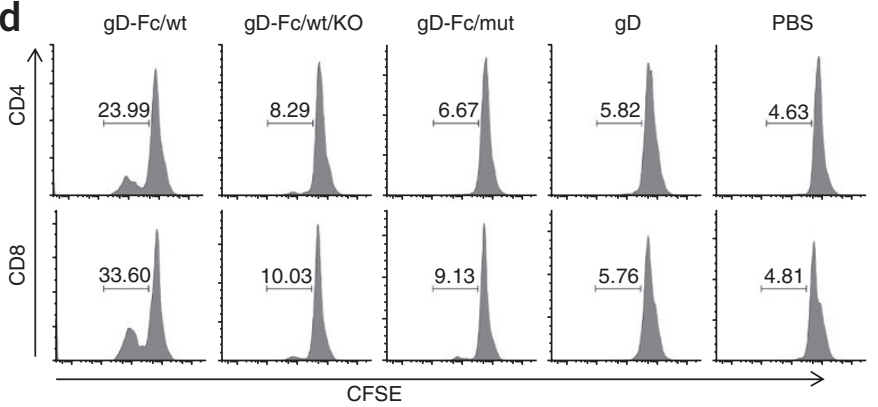

b
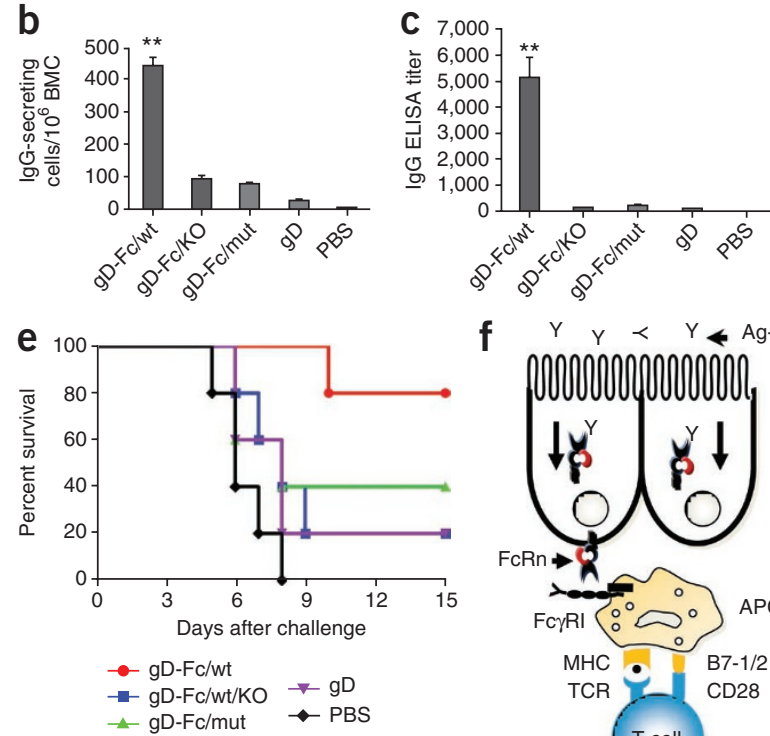

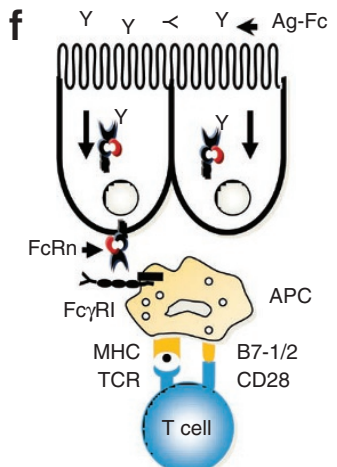

Figure 4 Increased memory immune response in FcRn-targeted mucosal immunization. (a) Induction of gD-specific memory B cells in the spleen. The frequency of gD-specific memory B cells was assessed 6 months after the boost. Memory B cells, defined as B220+ gD-surface ${ }^{+}$, were analyzed 6 months after the boost by FACS. Purified gD proteins were labeled with Alexa Fluro647. Spleen cells $\left(2 \times 10^{6}\right)$ were incubated with the $1 \mu \mathrm{g}$ Alexa Fluro647labeled gD proteins and B220 antibody. Numbers in the quadrants are the percentage of gD-specific memory B lymphocytes. (b) Long-lived HSV gDspecific antibody-secreting cells in the bone marrow. Bone marrow cells (BMC) removed 6 months after the boost were placed on gD-coated plates and quantified by ELISPOT analysis of IgG-secreting plasma cells. Data were pooled from two separate experiments with five mice in each experiment. The graphs were plotted based on the average ELISPOT for replicate wells. Values marked with asterisks are significantly greater $(P<0.01)$ from the gD-Fc/wt protein-immunized mice than those of other groups as indicated. (c) Durability of HSV-2 gD-specific serum IgG response. In two separate experiments, HSV-2 gD-specific IgG was quantified by ELISA in serum by endpoint titer from five mice at 6 months after the boost. HSV-specific IgG antibody was not detected in PBS-immunized mice. (d) Long-lived gD-specific T cell memory to FcRn-targeted mucosal vaccination. Spleen cells were isolated from the immunized mice 6 months after the boost, stained with CFSE and stimulated in vitro with $20 \mu \mathrm{g} / \mathrm{ml}$ of purified gD for $4 \mathrm{~d}$. Data are expressed in CFSE histograms of fluorescence intensity versus the number of fluorescing cells, indicating the percentage of the cell population positive for CD4 and $\mathrm{CD} 8$ antigen. Numbers in the quadrants are the percentage of $\mathrm{CD}^{+}$and $\mathrm{CD} 8^{+}$proliferating $\mathrm{T}$ cells. Representative flow cytometry profiles of two similar experiments with three mice per group are shown. Immunization conditions are displayed on the top. (e) Mean survival following genital HSV-2 challenge 6 months following the boost. The immunized mice were challenged intravaginally with $1 \times 10^{4}$ p.f.u. of HSV-2. Percentage of mice protected on the indicated days is calculated as the number of mice surviving divided by the number of mice in each group $(n=5)$. (f) Proposed model of FcRn-mediated mucosal vaccine delivery. The Fc-fused antigens are transported by FcRn and targeted to the mucosal antigen-presenting cells (APC), such as dendritic cells. Antigen is taken up by pinocytosis or FcrRI-mediated endocytosis in APCs, then processed and presented to T cells.

genital tract ${ }^{1,2,4}$. Perhaps intranasal immunization stimulates cells in the nasopharynx-associated lymphoid tissue and MeLNs, leading to the migration of antibody-secreting cells and $\mathrm{T}$ cells generated in the airway into the genital tract ${ }^{20}$. We intravaginally challenged immunized mice with a lethal dose of HSV-2 186 strain 4 weeks after the boost. All FcRn WT mice ( $n=15)$ immunized with $\mathrm{gD}$-Fc/wt survived with no obvious symptoms (Supplementary Fig. 7a), whereas gDFc/wt-immunized FcRn knockout mice $(n=15)$ were incompletely protected (Fig. 3a). These data indicate that full protection is dependent on FcRn. Additionally, virus titers measured in the vaginal washings showed that the $\mathrm{gD}-\mathrm{Fc} /$ wt-immunized mice had eliminated the virus by day 4 after challenge (Fig. 3b), whereas substantial viral levels remained in the other groups of mice through day 6 .

Several immune mechanisms may account for the protection of the distal vaginal mucosa. First, both mucosal and systemic antibody responses may play an important protective role. The sera passively transferred from the gD-Fc-immunized mice conferred a high level of protection (Supplementary Fig. 3) and a considerable amount of gD-specific IgG appeared in the vaginal secretions (Supplementary Fig. 6). After immunization with attenuated HSV-2, IgG contributes the majority of protection in mouse vaginal secretions ${ }^{20}$. Second, the presence of T lymphocytes in the vaginal epithelium of
HSV-2-challenged mice coincided with virus clearance. IFN- $\gamma$ is clearly indispensable for resistance to HSV-2 infections ${ }^{21}$. The $\mathrm{gD}-\mathrm{Fc} / \mathrm{wt}$ protein induced a significantly higher frequency of IFN- $\gamma$-producing $\mathrm{CD}^{+}$and $\mathrm{CD} 8^{+} \mathrm{T}$ cells in the vaginal tissues from the challenged mice (Fig. 3c). Overall these results demonstrate that FcRn-targeted mucosal immunization efficiently induced protective immunity.

Our studies further suggested that FcRn-targeted mucosal delivery of vaccine engendered an effective memory immune response. Immunological memory ${ }^{22,23}$ has been a concern in protein-based subunit mucosal vaccine development because although preparations frequently elicit immunity immediately after vaccination, protection decreases rapidly. A striking feature of our study is that FcRn-targeted mucosal immunization promoted and sustained high levels of gDspecific plasma cells and memory B and T cells. First, gD-Fc/wtimmunized mice developed considerably higher numbers of splenic memory B cells responsive to gD stimulation 6 months after the boost (Fig. 4a). Using enzyme-linked immunosorbent spot (ELISPOT), we also found the significantly $(P<0.01)$ higher number of gD-specific, IgG-secreting plasma cells in the bone marrow of mice immunized with gD-Fc/wt (Fig. 4b and Supplementary Fig. 8). It remains to be determined if gD-specific, IgA-secreting plasma cells also develop. 
Second, higher titers of gD-specific IgG were maintained for 6 months after the boost in the mice immunized with the gD-Fc/wt (Fig. 4c). Both the increase in germinal center and memory $\mathrm{B}$ cells in the spleen and the existence of long-lived plasma cells in the bone marrow niche can account for the maintenance of high levels of $\mathrm{gD}$-specific IgG in circulation. Third, we detected large numbers of CD4+ (Fig. 4d) and $\mathrm{CD}^{+}$(Fig. 4d) memory T-cell proliferation by carboxyfluorescein diacetate succinimidyl ester (CFSE) in response to gD recall in the mice immunized with $\mathrm{gD}-\mathrm{Fc} / \mathrm{wt}$, but not in any of the other groups. This result was also verified by considerably increased numbers of actively proliferating T cells over time (Supplementary Fig. 9). These data indicate that the $\mathrm{gD}$-specific $\mathrm{T}$ cells had maintained proliferative potential at least 6 months after the boost. Although the reason for the high memory T-cell activity is not completely clear, IL-2-producing $\mathrm{T}$ cells formed in the responding T-cell population (Supplementary Fig. 10) may be important because IL-2 is important in the successful long-term survival of memory $\mathrm{T}$ cells in vivo ${ }^{24}$. Fourth, to test if the memory immune response provided protection, we again intravaginally challenged the immunized mice with HSV-2 186 strain 6 months after the boost. Mice immunized with the gD-Fc/wt proteins exhibited less severe disease symptoms (Supplementary Fig. 7b) and had $80 \%$ survival (Fig. 4e), whereas the majority of mice in other groups succumbed to lethal mucosal infection.

Overall, our study shows that the FcRn/IgG transport pathway can be exploited to greatly enhance the efficacy of mucosally administered vaccines. Previous studies have taken advantage of Fc fusion proteins to augment the T-cell immune response to myelin basic protein ${ }^{25}$, and mucosally administered inactivated Francisella tularensis live vaccine strain/antibody immune complexes have been shown to enhance protection against a highly virulent strain ${ }^{26}$. We have shown that FcRn-targeted mucosal immunization differs considerably between WT and FcRn knockout mice or the gD-Fc/wt- and the gD$\mathrm{Fc} /$ mut-immunized mice in terms of mucosal and systemic immune responses, cytokine expression profiles, the maintenance of $\mathrm{T}$ - and B-cell memory and long-lived bone marrow plasma cells, and resistance to infection. We establish this principle using a modified form of the mouse IgG2a Fc fragment to facilitate the vaccine antigen delivery across the mucosal barrier. Earlier studies have shown intranasal and intravaginal immunization with $\mathrm{gD}$ or $\mathrm{gB}$ proteins in combination with $\mathrm{CpG}$ elicited immune responses and conferred partial protection to subsequent vaginal challenge with HSV-2 (refs. 27-30). The mechanism by which 'plain' gD or gB proteins crossed the airway or female genital epithelial barrier in those studies is not clear. The treatment of mice with agents, such as volatile anesthetics halothane and isoflurane or $0.5 \%$ Tween $^{27-30}$, could compromise the integrity of the alveolo-capillary or mucosal epithelial barrier ${ }^{31,32}$. Moreover, injections with Depo-Provera before vaginal immunization ${ }^{28-30}$ may affect the integrity of cell junctions between the vaginal epithelial cells ${ }^{4}$. In agreement with a previous report ${ }^{33}$, our other studies on examinations of the effect of $\mathrm{CpG}$ on protein transport across the nasal/tracheal mucosa (unpublished data) are not consistent with an affect of $\mathrm{CpG}$ on mucosal permeability or passive transfer. Our results document the benefits of FcRn targeting to maximize the potential of mucosally administered vaccines to counteract mucosal pathogens.

We suggest the following model for FcRn-targeted immunization (Fig. 4f). In general, mucosal dendritic cells capture antigens in mucosal-associated lymphoid tissues, and migrate to draining lymph nodes where they can activate $\mathrm{T}$ cells ${ }^{34,35}$ and initiate the cognate B-cell response. Persistence of vaccine antigens can facilitate longterm memory immune responses ${ }^{22,23}$. Thus, although FcRn-mediated transport is necessary for effective mucosal immunization, the ability of FcRn to protect $\mathrm{gD}-\mathrm{Fc} / \mathrm{wt}$ proteins from degradation may further support the development of systemic immunity by increasing the persistence of $\mathrm{gD}-\mathrm{Fc} / \mathrm{wt}$ in circulation. Furthermore, this same protection property may augment long-term humoral immunity by maintaining high levels of systemic IgG antibodies specific for $\mathrm{gD}-\mathrm{Fc} / \mathrm{wt}$. It remains to be determined whether this delivery method can augment preexisting immunity. Our findings suggest that FcRn-targeted mucosal immunization may be an effective strategy for maximizing the efficacy of vaccinations directed against a broad range of mucosal pathogens.

\section{METHODS}

Methods and any associated references are available in the online version of the paper at http://www.nature.com/naturebiotechnology/.

Note: Supplementary information is available on the Nature Biotechnology website.

\section{ACKNOWLEDGMENTS}

We thank K. Rosenthal and A. Harandi for their discussions of mucosal antigen transport and immunization. We thank G.J. Letchworth for helpful discussions and critical reading of the manuscript. We are grateful to N.E. Simister, Brandeis University, for supplying us with inner medullary collecting duct-FcRn cell line. We acknowledge the receipt of HSV-2 186 strain from L.R. Stanberry, Columbia University, and HSV gD plasmids from P. Spear, Northwestern University. We also acknowledge the helpful discussions with D. Mosser, D. Perez, S. Samal and W. Song. We are most grateful for the technical help from Y. Wang, L. Lu, X. Liu, S. Palaniyandi and Z. Li. This work was supported in part by the National Institutes of Health grants AI65892, AI67965, AI73139 (to X.Z.), DK56597 (to D.C.R.) and MAES competitive grants from the University of Maryland (to X.Z.).

\section{AUTHOR CONTRIBUTIONS}

L.Y. and X.Z. designed and performed experiments, analyzed data and wrote the paper. R.Z. and Y.B. conducted experiments. D.C.R. interpreted data and made editorial suggestions.

\section{COMPETING FINANCIAL INTERESTS}

The authors declare no competing financial interests.

Published online at http://www.nature.com/naturebiotechnology/.

Reprints and permissions information is available online at http://npg.nature.com/ reprintsandpermissions/.

1. Neutra, M.R. \& Kozlowski, P.A. Mucosal vaccines: the promise and the challenge Nat. Rev. Immunol. 6, 148-158 (2006).

2. Holmgren, J. \& Czerkinsky, C. Mucosal immunity and vaccines. Nat. Med. 11 Suppl S45-S53 (2005).

3. McGhee, J.R. et al. The mucosal immune system: from fundamental concepts to vaccine development. Vaccine 10, 75-88 (1992).

4. Gallichan, W.S. \& Rosenthal, K.L. Long-term immunity and protection against herpes simplex virus type 2 in the murine female genital tract after mucosal but not systemic immunization. J. Infect. Dis. 177, 1155-1161 (1998).

5. Neutra, M.R., Mantis, N.J. \& Kraehenbuhl, J.-P. Collaboration of epithelial cells with organized mucosal lymphoid tissues. Nat. Immunol. 2, 1004-1009 (2001).

6. Nochi, T. et al. A novel M cell-specific carbohydrate-targeted mucosal vaccine effectively induces antigen-specific immune responses. J. Exp. Med. 204, 2789-2796 (2007).

7. Ghetie, V. \& Ward, E.S. Multiple roles for the major histocompatibility complex class I-related receptor FcRn. Annu. Rev. Immunol. 18, 739-766 (2000).

8. He, W. et al. FcRn-mediated antibody transport across epithelial cells revealed by electron tomography. Nature 455, 542-546 (2008).

9. Dickinson, B.L. et al. Bidirectional FcRn-dependent IgG transport in a polarized human intestinal epithelial cell line. J. Clin. Invest. 104, 903-911 (1999).

10. Roopenian, D.C. \& Akilesh, S. FcRn: the neonatal Fc receptor comes of age. Nat. Rev. Immunol. 7, 715-725 (2007).

11. Baker, K. et al. Immune and non-immune functions of the (not so) neonatal Fc receptor, FcRn. Semin. Immunopathol. 31, 223-236 (2009).

12. Yoshida, M. et al. Neonatal Fc receptor for IgG regulates mucosal immune responses to luminal bacteria. J. Clin. Invest. 116, 2142-2151 (2006).

13. Kim, J.K., Tsen, M.F., Ghetie, V. \& Ward, E.S. Localization of the site of the murine IgG1 molecule that is involved in binding to the murine intestinal Fc receptor. Eur. J. Immunol. 24, 2429-2434 (1994).

14. Duncan, A.R. \& Winter, G. The binding site for C1q on IgG. Nature 332, 738-740 (1988).

15. McCarthy, K.M., Yoong, Y. \& Simister, N.E. Bidirectional transcytosis of IgG by the rat neonatal Fc receptor expressed in a rat kidney cell line: a system to study protein transport across epithelia. J. Cell Sci. 113, 1277-1285 (2000). 
16. Roopenian, D.C. et al. The MHC class I-like IgG receptor controls perinatal IgG transport, IgG homeostasis, and fate of IgG-Fc-coupled drugs. J. Immunol. 170, 3528-3533 (2003).

17. van Duin, D., Medzhitov, R. \& Shaw, A.C. Triggering TLR signaling in vaccination. Trends Immunol. 27, 49-55 (2006).

18. Wolf, A.J. et al. Initiation of the adaptive immune response to Mycobacterium tuberculosis depends on antigen production in the local lymph node, not the lungs. J. Exp. Med. 205, 105-115 (2008).

19. Moyron-Quiroz, J.E. et al. Role of inducible bronchus associated lymphoid tissue (iBALT) in respiratory immunity. Nat. Med. 10, 927-934 (2004).

20. Parr, E.L. \& Parr, M.B. Immunoglobulin G is the main protective antibody in mouse vaginal secretions after vaginal immunization with attenuated herpes simplex virus type 2. J. Virol. 71, 8109-8115 (1997).

21. Milligan, G.N., Dudley-McClain, K.L., Chu, C.F. \& Young, C.G. Efficacy of genital T cell responses to herpes simplex virus type 2 resulting from immunization of the nasal mucosa. Virology 318, 507-515 (2004).

22. Ahmed, R. \& Gray, D. Immunological memory and protective immunity: understanding their relation. Science 272, 54-60 (1996).

23. Bernasconi, N.L., Traggiai, E. \& Lanzavecchia, A. Maintenance of serological memory by polyclonal activation of human memory B cells. Science 298, 2199-2202 (2002).

24. Dooms, H., Wolslegel, K., Lin, P. \& Abbas, A.K. Interleukin-2 enhances CD4 ${ }^{+}$ $\mathrm{T}$ cell memory by promoting the generation of IL-7R $\alpha$-expressing cells. J. Exp. Med. 204, 547-557 (2007).

25. Mi, W. et al. Targeting the neonatal Fc receptor for antigen delivery using engineered Fc fragments. J. Immunol. 181, 7550-7561 (2008).

26. Rawool, D.B. et al. Utilization of Fc receptors as a mucosal vaccine strategy against an intracellular bacterium, Francisella tularensis. J. Immunol. 180, 5548-5557 (2008).
27. Gallichan, W.S et al. Intranasal immunization with CpG oligodeoxynucleotides as an adjuvant dramatically increases IgA and protection against herpes simplex virus-2 in the genital tract. J. Immunol. 166, 3451-3457 (2001).

28. Kwant, A. \& Rosenthal, K.L. Intravaginal immunization with viral subunit protein plus $\mathrm{CpG}$ oligodeoxynucleotides induces protective immunity against HSV-2. Vaccine $\mathbf{2 2}$ 3098-3104 (2004).

29. Tengvall, S., Lundqvist, A., Eisenberg, R.J., Cohen, G.H. \& Harandi, A.M. Mucosal administration of $\mathrm{CpG}$ oligodeoxynucleotide elicits strong $\mathrm{CC}$ and $\mathrm{CXC}$ chemokine responses in the vagina and serves as a potent Th1-tilting adjuvant for recombinant gD2 protein vaccination against genital herpes. J. Virol. 80, 5283-5291 (2006)

30. Lindqvist, M., Persson, J., Thörn, K. \& Harandi, A.M. The mucosal adjuvant effect of alpha-galactosylceramide for induction of protective immunity to sexually transmitted viral infection. J. Immunol. 182, 6435-6443 (2009).

31. ChangLai, S.P., Hung, W.T. \& Liao, K.K. Detecting alveolar epithelial injury following volatile anesthetics by $(99 \mathrm{~m})$ Tc DTPA radioaerosol inhalation lung scan. Respiration 66 506-510 (1999).

32. Lin, $\mathrm{H}$. et al. Enhancing effect of surfactants on fexofenadine. $\mathrm{HCl}$ transport across the human nasal epithelial cell monolayer. Int. J. Pharm. 330, 23-31 (2007).

33. Kodama, S., Abe, N., Hirano, T. \& Suzuki, M. Safety and efficacy of nasal application of $\mathrm{CpG}$ oligodeoxynucleotide as a mucosal adjuvant. Laryngoscope 116, 331-335 (2006).

34. Kelsall, B.L. \& Rescigno, M. Mucosal dendritic cells in immunity and inflammation. Nat. Immunol. 5, 1091-1095 (2004).

35. Yoshida, M. et al. Human neonatal Fc receptor mediates transport of IgG into luminal secretions for delivery of antigens to mucosal dendritic cells. Immunity $\mathbf{2 0}$ 769-783 (2004). 


\section{ONLINE METHODS}

Cells, antibodies and virus. Inner medullary collecting duct (IMCD) cell line and IMCD cells expressing rat FcRn were obtained from Neil Simister. Vero and Chinese hamster ovary $(\mathrm{CHO}-\mathrm{K})$ cells were purchased from American Tissue Culture Collection (ATCC). IMCD, Vero and CHO cells were maintained in DMEM complete medium (Invitrogen Life Technologies) supplemented with $10 \mathrm{mM}$ HEPES, 10\% FBS, $2 \mathrm{mM}$ L-glutamine, nonessential amino acids, and penicillin $(0.1 \mu \mathrm{g} / \mathrm{ml}) /$ streptomycin $(0.292 \mu \mathrm{g} / \mathrm{ml})$. Recombinant IMCD and CHO cells were grown in $400 \mu \mathrm{g} / \mathrm{ml}$ of G418, when necessary. Cells from spleen or bone marrow were grown in complete RPMI 1640 medium. HSV-2 strain 186 was from Lawrence Stanberry and HSV-2 stocks were prepared by infection of Vero cell monolayers at a multiplicity of infection (MOI) of 0.01. All cells and viruses were grown in a humidified atmosphere of $5 \% \mathrm{CO}_{2}$ at $37^{\circ} \mathrm{C}$. Affinity-purified antibody for mouse $\mathrm{FcRn}$ was made as previously described ${ }^{30}$. Horseradish peroxidase (HRP)-conjugated donkey antirabbit or rabbit anti-mouse antibody was purchased from Pierce (Rockland). Purified mouse IgG and chicken IgY was from Rockland Laboratories, and HRP-conjugated goat anti-mouse IgG1, IgG2a and IgG3 were from Southern Biotech. All DNA-modifying enzymes were purchased from New England Biolabs. Purified HSV-2 glycoprotein D was purchased from Meridian Life Science.

Expression of $\mathrm{gD}-\mathrm{Fc}$ fusion proteins. The cDNA encoding the extracellular domain of HSV-2 gD (26-340 aa) was amplified by PCR from a plasmid provided by Patricia G. Spear using the primer pair (5'-CCCAAGCTTACCA TGGGGCGTTTGACCTCCGGCGTC-3' 5' $^{\prime}$-AGATCCCGAGCCACCTCCT CCGGACCCACCCCCGCCTGATCCGCCCGGGTTGCTGGGGG-3'). The antisense primer introduces an extension with 14 codons for glycine and serine residues (GSGGGGSGGGGSGS). The Fc-fragment of mouse IgG2a containing the hinge, an extended $\mathrm{CH} 2$ and a $\mathrm{CH} 3$ domain was amplified by RT-PCR from the OKT3 hybridoma. The mouse IgG2a Fc fragment was used because mouse IgG2a, but not IgG1, is capable of binding mouse Fc $\gamma R I$, a high-affinity receptor for IgG. Similarly, the forward primer for IgG2a Fc has complementary glycine and serine codons for gD. A mutant Fc (HQ310 and HN433), unable to bind mouse FcRn, was made by oligonucleotide site-directed mutagenesis (Clontech) and designated as an Fc/mut. To construct a nonlytic Fc fragment, oligonucleotide site-directed mutagenesis was used to replace the $\mathrm{C} 1 \mathrm{q}$ binding motif Glu318, Lys320, Lys322 with Ala residues ${ }^{14}$. Fusions were then performed in a PCR-based gene assembly approach by mixing the cDNA for $\mathrm{gD}$ and the Fc fragment. All these DNA fragments were ligated into the pCDNA3 vector. Each construct was verified by DNA sequencing.

The plasmids containing the chimeric $\mathrm{gD}-\mathrm{Fc}$ fragment were transfected into CHO cells. G418-resistant clones were selected for secretion of gD-Fc fusion protein. SDS-PAGE and western blot analysis were performed to assess the recombinant fusion proteins in serum-free medium (Invitrogen). The highest secreting clones were screened. Recombinant proteins were purified from $\mathrm{CHO}$ cell supernatants by affinity chromatography using Protein A Sepharose 4 Fast Flow (Amersham) or goat anti-mouse IgG affinity column (Rockland). Protein concentration was measured with Coomassie (Bradford) protein assay kit (Pierce) using mouse IgG2a as standard.

Western blot analysis and SDS-PAGE gel electrophoresis. The proteins or cell lysates were resolved on a 12\% SDS-PAGE gel under reducing or nonreducing conditions. Proteins were transferred onto a nitrocellulose membrane (Schleicher \& Schuell). The membranes were blocked with 5\% nonfat milk, probed separately with anti-gD, anti-IgG Fc antibody or anti-mouse FcRn for $1 \mathrm{~h}$, and followed by incubation with HRP-conjugated rabbit anti-mouse or donkey anti-rabbit antibodies. All blocking, incubation and washing were performed in PBST solution (PBS and 0.05\% Tween 20). Proteins were visualized by enhanced chemiluminescence (ECL) (Pierce).

In vitro and in vivo transcytosis. The in vitro $\mathrm{IgG}$ transport assay was performed as a modification of previously described methods ${ }^{15,36}$. IMCD cells expressing rat FcRn were grown on transwell filter inserts (Corning Costar) to form a monolayer exhibiting transepithelial electrical resistances (TER, $300 \Omega . \mathrm{cm}^{2}$ ). TER was measured using a tissue-resistance measurement equipped with planar electrodes (World Precision Instruments). Monolayers were equilibrated in Hanks' balanced salt solution. Fusion proteins $(50 \mu \mathrm{g} / \mathrm{ml})$ were applied to the apical compartment, and incubated with DMEM medium supplied with or without $1 \mathrm{mg} / \mathrm{ml}$ of mouse IgG or chicken IgY as competitors for $2 \mathrm{~h}$ at $37^{\circ} \mathrm{C}$ degree. Transported proteins were sampled from the basolateral chamber and analyzed by reducing SDS-PAGE and western blot-ECL. For in vivo transport, the biotinylated $20 \mu \mathrm{g}$ of fusion proteins or $\mathrm{gD}$ alone in $20 \mu \mathrm{l}$ of PBS were administered i.n. into the mice that were anesthetized with $100 \mu \mathrm{l}$ of avertin $(40 \mathrm{mg} / \mathrm{ml})$. At indicated time points or $8 \mathrm{~h}$ later, transported proteins in sera were determined by ELISA.

Mouse immunization and virus challenge. Six- to eight-week-old female inbred C57BL/6 mice were purchased from Charles River. FcRn knockout mice on a C57BL/6 background ${ }^{16}$ were from the Jackson Laboratory. All mice were housed in the animal resources facility at the University of Maryland. All animal studies were reviewed and approved by the Institutional Animal Care and Use Committee. All proteins and PBS were mixed with immunostimulatory DNA rich in CG motifs CpG oligonucleotide 1826 (abbreviated CpG) to overcome possible mucosal immune tolerance ${ }^{37}$. Groups of five mice were immunized i.n. with $20 \mu \mathrm{l}$ of $20 \mu \mathrm{g} \mathrm{gD}-\mathrm{Fc} / \mathrm{wt}, \mathrm{gD}-\mathrm{Fc} / \mathrm{mut}$, or recombinant $\mathrm{gD}$ alone or in combination with $20 \mu \mathrm{g}$ CpG (5'-TCCATGACGTTCCTGACG TT- $3^{\prime}$ ) (Invitrogen) per immunization at weeks 0 and 2 with an intraperitoneal injection of $100 \mu \mathrm{l}$ of avertin $(40 \mathrm{mg} / \mathrm{ml})$. An additional group of five mice was mock-immunized with PBS following the same schedule. Mice were kept on their backs under anesthesia to allow the inoculum to be taken up.

Mice were inoculated with viruses intravaginally as described previously ${ }^{27,38}$. Briefly, each mouse was subcutaneously treated with $3 \mathrm{mg}$ of medroxyprogesterone acetate (Depo-Provera) $10 \mathrm{~d}$ before virus inoculation. Hormonal pretreatment was necessary to induce susceptibility of mice to genital HSV-2 inoculation, which may cause thinning of the genital epithelium or induction of the HSV entry receptor, nectin-1, on vaginal epithelial cells. Mice anesthetized by avertin $(40 \mathrm{mg} / \mathrm{ml}$, Sigma) were infected intravaginally with $1 \times$ $10^{4}$ plaque-forming unit (p.f.u.)/100 $\mu \mathrm{l}$ of HSV-2 strain 186. Mice were kept on their backs under the influence of anesthesia for $45 \mathrm{~min}$ to allow infection. Mice were monitored for $15 \mathrm{~d}$ for disease and death. Mice exhibiting severe disease symptom were euthanized. For virus titration, virus was inoculated into Vero cells and incubated for $45 \mathrm{~min}$ at $37^{\circ} \mathrm{C}$. The cells were washed and DMEM containing $0.8 \%$ methylcellulose and $2 \%$ FBS was added to overlay the cells. The cells were cultured for $3 \mathrm{~d}$, the overlay was removed, and the cells were fixed with $3.7 \%$ formaldehyde for $1 \mathrm{~h}$, and stained with $1 \%$ crystal violet.

Preparation of single-cell suspensions from lymph nodes, spleen, lung and vaginal tissues. Spleens and lymph nodes ${ }^{39}$ were made into single-cell suspensions by passage through a sterile mesh screen. Cells were resuspended in Hanks' balanced salt solution (HBSS) and counted by trypan blue dye exclusion. For each experiment, cells were generally pooled from 3-5 mice in each group. For preparation of single-cell suspensions from the lung, mice were anesthetized with $400 \mu \mathrm{l}$ of avertin $(40 \mathrm{mg} / \mathrm{ml})$ by intraperitoneal injection. Lungs were perfused with $10 \mathrm{ml}$ PBS through the right ventricle, removed, minced with blades, and incubated with HBSS containing $2.5 \mathrm{mM}$ HEPES and $1.3 \mathrm{mM}$ EDTA at $37^{\circ} \mathrm{C}$ for $30 \mathrm{~min}$, followed by treatment at $37^{\circ} \mathrm{C}$ for $1 \mathrm{~h}$ with $2.5 \mathrm{mg} / \mathrm{ml}$ collagenase D (Roche) in RPMI 1640 medium containing 5\% FBS The resulting cells were filtered through a $70-\mu \mathrm{m}$ cell strainer (BD Biosciences) and used for fluorescence-activated cell sorting (FACS) analysis.

For isolation of vaginal cells, the vagina was excised, cut longitudinally and minced with a sterile scalpel in complete RPMI 1640 culture medium. Minced tissues (epithelium and lamina propria) were digested in complete medium with sterile $0.25 \%$ collagenase D (Sigma). Digestion was accomplished with shaking incubation at $37^{\circ} \mathrm{C}$ for $30 \mathrm{~min}$. After digestion, tissues and cells were filtered through a sterile gauze mesh and washed with RPMI 1640 medium, and additional tissue debris was excluded by slow-speed centrifugation for $1 \mathrm{~min}$. Cells were collected from the supernatant by centrifugation, resuspended in HBSS and counted by trypan blue dye exclusion.

Flow cytometry. Single-cell suspensions from the spleen, lung or vaginal tissues were collected and cells were spun down. Erythrocytes were then lysed in $0.14 \mathrm{M} \mathrm{NH}_{4} \mathrm{Cl}, 0.017 \mathrm{M}$ Tris- $\mathrm{HCl}$ at $\mathrm{pH} 7.2$ on ice for $5 \mathrm{~min}$. 
Cells were preincubated with an Fc block (monoclonal antibody (mAb) to CD16-CD32, 2.4G2, PharMingen) and washed in FACS buffer (HBSS, $2 \%$ BSA, $0.01 \%$ sodium azide). Cells were incubated with specific antibody $\left(0.25 \mu \mathrm{g} / 10^{6}\right.$ cells $\left./ 100 \mu \mathrm{l}\right)$ directly conjugated to fluorescein isothiocyanate (FITC), phycoerythrin, washed, transferred to FACS buffer and analyzed using a FACSAire (Becton Dickinson) and FlowJo software (Tree Star). The mAbs (PharMingen) we used were anti-CD3e, 500A2; anti-CD4, RM4- 5; anti-CD8, 53-6.7; anti-IFN- $\gamma$, XMG1.2; anti-B220, RA3-6B2; FAS, Jo2; CD19, 1D3. PNA was from Sigma. Purified HSV-2 gD proteins were labeled with Alexa Fluor 647 protein labeling kit (Invitrogen) according to the manufacturer's instructions. Cells incubated with isotype control antibodies were used to determine the background fluorescence. The isotype control antibodies included in each experiment were considered the true baseline fluorescence used to evaluate and illustrate the results for the cell-specific antigen markers.

T-cell proliferation. Single-cell suspensions from mouse spleen were suspended in RPMI- 1640 with $1 \%$ FCS, $2.5 \mathrm{mM} \mathrm{HEPES} \mathrm{at} 10^{7} / \mathrm{ml}$. Carboxyfluorescein diacetate succinimidyl ester (CFSE, $5 \mathrm{mM}$ stock, Invitrogen) was diluted tenfold with PBS, $4 \mu \mathrm{l}$ of diluted CFSE was then added into $10^{7} / \mathrm{ml}$ cells for a $2 \mu \mathrm{M}$ final concentration. The reaction was incubated for $10 \mathrm{~min}$ at $37^{\circ} \mathrm{C}$. Cold FBS ( $1 \mathrm{ml})$ was added and incubated on ice for $5 \mathrm{~min}$ to stop the reaction. The cells were washed twice with RPMI-1640 with $10 \%$ FCS. Labeled cells $\left(5 \times 10^{5}\right)$ were plated into 96 -well plates in $200 \mu \mathrm{l}$ of medium and cultured for $5 \mathrm{~d}$. The cells were then harvested and subjected to flow cytometry assay.

Intracellular cytokine staining. Intracellular IFN $-\gamma$ production by primed $\mathrm{CD}^{+}$and $\mathrm{CD} 8^{+} \mathrm{T}$ cells was evaluated using bulk splenocytes or isolated lung or vaginal infiltrating lymphocytes incubated for $4 \mathrm{~h}$ with $25 \mu \mathrm{g} / \mathrm{ml}$ of the purified $\mathrm{gD}$ protein or medium alone. Cells were then cultured for another $6 \mathrm{~h}$ in the presence of brefeldin A (Sigma). The cells were washed and incubated with anti-CD16/CD32 antibody to block Fc $\gamma$ receptors, and stained with antimouse CD4, CD8 and CD3 antibodies for $15 \mathrm{~min}$ at $4{ }^{\circ} \mathrm{C}$. After fixation and membrane penetration with Cytofix/Cytoperm Plus (BD Biosciences), cells were stained for intracellular IFN- $\gamma$ for $30 \mathrm{~min}$ on ice. Cells were washed three times, resuspended in FACS buffer and analyzed by flow cytometry.

ELISA, ELISPOT and neutralization test. For the detection of gD-specific antibodies in serum, bronchial lavage and vaginal fluid, high-binding ELISA plates (Maxisorp, Nunc) were coated with $5 \mu \mathrm{g} / \mathrm{ml}$ of recombinant gD protein in PBS and incubated overnight at $4{ }^{\circ} \mathrm{C}$. Plates were then washed three times with $0.02 \%$ Tween 20 in PBS and blocked with $1 \%$ BSA in PBS for $1 \mathrm{~h}$ at $25{ }^{\circ} \mathrm{C}$. Samples were serially diluted in $0.25 \%$ BSA-PBS and incubated for $2 \mathrm{~h}$ at $25^{\circ} \mathrm{C}$. HRP-conjugated rabbit anti-mouse IgG antibody $(1: 2,000$, Pierce) or anti-mouse subclass-specific antibodies (1:5,000, Southern Biotech) was added and followed by colorimetric assay using substrate tetramethylbenzidine and a Victor III microplate reader (PerkinElmer). Titers represent the highest dilution of samples showing a twofold $\mathrm{OD}_{450}$ value over controls. Neutralizing antibodies were measured by a standard virus neutralization assay. Sera were heat-inactivated, diluted first tenfold, then in twofold steps in MEM with 2\% FBS. Fifty p.f.u. of HSV-2 was added per well and incubated at $37^{\circ} \mathrm{C}$ for $1 \mathrm{~h}$. Plaque assays were done. The titers were expressed as the reciprocal of the twofold serial dilution preventing the appearance of the cytopathic effects. Each assay was done in triplicate.

For measuring gD-specific, antibody-producing plasma cells, the 96-well ELISPOT plates (Millipore) were coated with $5 \mu \mathrm{g} / \mathrm{ml} \mathrm{gD}$ and blocked with RPMI 5\% FCS (Invitrogen) for $90 \mathrm{~min}$ at $37^{\circ} \mathrm{C}$ and $5 \% \mathrm{CO}_{2}$. Serial dilutions of bone marrow single-cell suspensions were prepared in RPMI and incubated in the coated wells for $24 \mathrm{~h}$ at $37^{\circ} \mathrm{C}$ in $5 \% \mathrm{CO}_{2}$. Cells were removed, and plates were washed 5 times with $0.1 \%$ Tween 20 in PBS, then incubated with biotin-labeled goat anti-mouse, IgG-specific antibody (1:1,500, Sigma) for $2 \mathrm{~h}$. After washing the cells, with PBS, the avidin-conjugated HRP $(1: 2,000$, Vector Laboratories) was added and incubated for $1 \mathrm{~h}$, followed by substrate from the AEC kit (BD Biosciences). Spots were counted with ELISPOT Reader and analyzed with software (Zeiss). Mouse cytokines IFN- $\gamma$, IL-2 and IL-4 from the cell culture supernatant were analyzed by ELISA according to the manufacturer's instructions (BD Biosciences).

Immunofluorescence. Immunofluorescence was performed as previously described ${ }^{40}$. Briefly, frozen serial sections of tissues were cold-fixed in acetone for $3 \mathrm{~min}$ at $4{ }^{\circ} \mathrm{C}$ and blocked with $10 \%$ normal goat serum and stained with biotin-PNA (germinal centers, red), Alexa 647-IgD or antiB220 (B cells, green), followed by Alexa 555-avidin or 488 Fluor-conjugated $\mathrm{IgG}$ of the corresponding species. After each step, cells were washed at least three times with $0.1 \%$ Tween-20 in PBS. Cover slips were mounted on slides with Prolong TM intifada kit (Molecular Probes) and examined using a Zeiss LSM 510 confocal fluorescence microscopy. Images were processed by Adobe Photoshop 7.0.

Passive transfer of immune sera. Sera were collected from 3-5 mice per group 4 weeks after the immunization, then pooled, heat inactivated and stored frozen at $-80{ }^{\circ} \mathrm{C}$ until use. Mice received a single intraperitoneal injection of $0.3 \mathrm{ml}$ immune sera $3 \mathrm{~d}$ before challenge to allow distribution and equilibration of antibody to all tissues before virus inoculation. Mice were challenged intravaginally with $1 \times 10^{4}$ p.f.u. HSV-2 186 strain.

Statistics analysis. To compare survival curves, we used Kaplan-Meier logrank analyses. Antibody titers, serum gD concentration, cytokine concentration and virus titers were assessed by using the unpaired two-tailed $t$-test. Graph Pad Prism 5 provided the software for the statistical analysis.

36. Liu, X. et al. Activation of the JAK/STAT-1 signaling pathway by IFN-gamma can down-regulate functional expression of the MHC class I-related neonatal Fc receptor for IgG. J. Immunol. 181, 449-463 (2008).

37. Mestecky, J., Russell, J.N.W. \& Elson, C.O. Perspectives on mucosal vaccines: is mucosal tolerance a barrier? J. Immunol. 179, 5633-5638 (2007).

38. Milligan, G.N., Bernstein, D.I. \& Bourne, N. T lymphocytes are required for protection of the vaginal mucosae and sensory ganglia of immune mice against reinfection with herpes simplex virus type 2. J. Immunol. 160, 6093-6100 (1998).

39. Van den Broeck, W., Derore, A. \& Simoens, P. Anatomy and nomenclature of murine lymph nodes: descriptive study and nomenclatory standardization in BALB/cAnNCr mice. J. Immunol. Methods 312, 12-19 (2006).

40. Ye, L. et al. The MHC class II-associated invariant chain interacts with the neonatal $\mathrm{Fc}$ gamma receptor and modulates its trafficking to endosomal/lysosomal compartments. J. Immunol. 181, 2572-2585 (2008). 